\title{
A Generalization of $\varphi$-conditional Expectation and Operator Valued Weight
}

By

\author{
Masataka HIRAKAWA*
}

\begin{abstract}
Let $M$ be a von Neumann algebra and $N$ a von Neumann subalgebra of $M$. For any n.f.s. weights $\varphi$ and $\psi$ on $M$ and $N$, respectively, we construct a normal map $E: M_{+} \rightarrow \hat{N}_{+}$, which is the $\varphi$-conditional expectation if $\psi=\left.\varphi\right|_{N}$, and is the operator valued weight if $\sigma_{t}^{\psi}=\left.\sigma_{t}^{\varphi}\right|_{N}(\forall t \in \mathbb{R})$.
\end{abstract}

\section{Introduction}

Let $M$ be a von Neumann algebra with a normal faithful semifinite (n.f.s.) weight $\varphi$, and $N$ be a von Neumann subalgebra of $M$ with an n.f.s. weight $\psi$. The conditional expectations or the operator valued weights from $M$ to $N$ have been studied by several authors.

In [7], Takesaki showed that there exists a faitheful normal norm 1 projection (which is also called a conditional expectation) $E$ which satisfies $\varphi=\varphi \circ E$ if and only if $\left.\varphi\right|_{N}$ is semifinite and $\sigma_{t}^{\varphi}(N)=N(\forall t \in \mathbf{R})$.

In [3], Haagerup showed that there exists an n.f.s. operator valued weight $E$ which satisfies $\varphi=\psi \circ E$ if and only if $\left.\sigma_{t}^{\varphi}\right|_{N}=\sigma_{t}^{\psi}(\forall t \in \mathbf{R})$.

In another direction, when $\psi=\left.\varphi\right|_{N}$, Accardi and Cecchini constructed in [1] the normal completely positive map $E$ which satisfies

$$
\begin{gathered}
\left(E\left(x_{1}^{*} x_{2}\right) J_{N} \eta_{\psi}\left(y_{1}\right) \mid J_{N} \eta_{\psi}\left(y_{2}\right)\right)=\left(y_{1}^{*} y_{2} J_{M} \eta_{\varphi}\left(x_{1}\right) \mid J_{M} \eta_{\varphi}\left(x_{2}\right)\right), \\
\forall x_{1}, x_{2} \in \mathbf{n}_{\varphi}, \quad \forall y_{1}, y_{2} \in \mathbf{n}_{\psi} .
\end{gathered}
$$

This map $E$ is called the $\varphi$-conditional expectation and is a norm 1 projection if $\varphi$ satisfies $\sigma_{t}^{\varphi}(N)=N \forall t \in \mathbb{R}$.

In this paper, we generalize this Accardi and Cecchini's construction to the case that $\psi$ is not necessarily equal to $\left.\varphi\right|_{N}$. And we show that if $\varphi$ and $\psi$ satisfy $\left.\sigma_{t}^{\varphi}\right|_{N}=\sigma_{t}^{\psi}, \forall t \in \mathbb{R}$, then the constructed map is the operator valued weight.

Communicated by H. Araki, April 8, 1991.

1991 Mathematics Subject Classifications: 46L50

* Research Institute for Mathematical Sciences, Kyoto University, Kyoto 606, Japan. 


\section{Notations}

Throughout this paper, $M$ will denote a von Neumann algebra and $N$ a von Neumann subalgebra of $M$. We also assume that $\left(M, \mathfrak{T}, J_{M}, \mathscr{P}_{M}\right)$ and $\left(N, \mathfrak{R}, J_{N}, \mathscr{P}_{N}\right)$ are standard forms and $\varphi$ and $\psi$ are n.f.s. weights on $M$ and $N$, respectively.

Then we can define the linear map $x \in \mathrm{n}_{\varphi} \mapsto \eta_{\varphi}(x) \in \mathbb{D}$ canonically, and the linear map $x \in \mathbb{m}_{\varphi} \mapsto \theta_{\varphi}(x) \in M_{*}$ as $\theta_{\varphi}\left(y^{*} z\right)=\omega_{J_{M} \eta_{\varphi}(y), J_{M} \eta_{\varphi}(z)}$ for $y, z \in \mathbb{R}_{\varphi}$. The map $\theta_{\varphi}(x)$ has the following properties.

$$
\begin{aligned}
& \text { (i) } 0 \leq x \Rightarrow 0 \leq \theta_{\varphi}(x) . \\
& \text { (ii) } f \in M_{*}^{+}, f \leq \varphi \Rightarrow f=\theta_{\varphi}(x) \quad\left(\exists x \in \mathrm{m}_{\varphi} \cap M_{+}\right) . \\
& \text {(iii) } \varphi(x)=\sup \left\{\left\langle x, \theta_{\varphi}(y)\right\rangle ; y \in \mathrm{m}_{\varphi} \cap M_{+},\|y\|<1\right\} \quad\left(\forall x \in M_{+}\right) .
\end{aligned}
$$

The analogous objects $\eta_{\psi}(y), \theta_{\psi}(y)$ for $N$ are defined with respect to $\psi$.

Let $\hat{N}_{+}$be the extended positive part of $N$ [3]. An $N$-weight on $M$ is a map $T: M_{+} \rightarrow \hat{N}_{+}$which satisfies the following conditions:

$$
\begin{aligned}
& \text { (i) } T(\lambda x)=\lambda T(x) \quad\left(\lambda \geq 0, x \in M_{+}\right) . \\
& \text {(ii) } T(x+y)=T(x)+T(y) \quad\left(x, y \in \mathbb{M}_{+}\right) .
\end{aligned}
$$

Moreover, we say that $T$ is an operator valued weight if

$$
\text { (iii) } T\left(a^{*} x a\right)=a^{*} T(x) a, \quad\left(x \in M_{+}, a \in N\right) \text {. }
$$

We put

$$
\begin{gathered}
\mathfrak{n}_{T}=\left\{x \in M ; T\left(x^{*} x\right) \in N_{+}\right\}, \\
\mathrm{m}_{T}=\mathfrak{n}_{T}^{*} \mathfrak{n}_{T}=\operatorname{span}\left\{x^{*} y ; x, y \in \mathfrak{n}_{T}\right\} .
\end{gathered}
$$

We say that $T$ is normal if

$$
x_{i} \nearrow x \Rightarrow T\left(x_{i}\right) \nearrow T(x) \quad\left(x_{i}, x \in M_{+}\right) .
$$

$T$ is faithful if $T\left(x^{*} x\right)=0$ implies $x=0$, and $T$ is semifinite if $m_{T}$ is $\sigma$-weakly dense in $M$.

\section{§1. A Generalization of $\varphi$-conditional Expectation}

For $\varphi$ and $\psi$, we put

$$
\begin{aligned}
\mathfrak{N} & =\mathfrak{N}_{\varphi, \psi} \\
& =\left\{a \in M ; \text { there exists } \lambda>0 \text { such that } \varphi\left(y^{*} a^{*} a y\right) \leq \lambda \psi\left(y^{*} y\right) \text { for any } y \in N\right\} . \\
\mathfrak{M} & =\mathfrak{M}_{\varphi, \psi}=\operatorname{span}\left\{b^{*} a \in M ; a, b \in \mathfrak{N}_{\varphi, \psi}\right\} .
\end{aligned}
$$

Since $\mathfrak{N}$ is a left ideal, there exists a projection $e=e_{\varphi, \psi} \in M$ such that

$$
\overline{\mathfrak{N}}^{\sigma-w}=M e_{\varphi, \psi} \text {. }
$$


Lemma 1.1. (i) $\mathfrak{N} N \subset \mathfrak{N}, N \mathfrak{M} N \subset \mathfrak{M}$.

(ii) $\mathfrak{N} \mathfrak{n}_{\psi} \subset \mathfrak{n}_{\varphi}, \mathfrak{n}_{\psi}^{*} \mathfrak{M} \mathfrak{n}_{\psi} \subset \mathfrak{m}_{\varphi}$.

(iii) $e_{\varphi, \psi} \in N^{\prime} \cap M$.

Proof. (i) and (ii) are immediate.

(iii) For each unitary $u \in N$ and each $x \in \mathfrak{M} \cap M^{+}, u^{*} x u \in \mathfrak{M} \cap M^{+}$from (i), hence we have $u^{*} e_{\varphi, \psi} u \leq e_{\varphi, \psi}$ and $u e_{\varphi, \psi} u^{*} \leq e_{\varphi, \psi}$. Therefore for each unitary $u \in N$, we have $u^{*} e_{\varphi, \psi} u=e_{\varphi, \psi}$, this implies $e_{\varphi, \psi} \in N^{\prime} \cap M$

Example 1.2. (i) If $\left.\varphi\right|_{N} \leq \lambda \psi$ for some $\lambda>0$, then $e_{\varphi, \psi}=1$.

(ii) If $\sigma_{t}^{\varphi}(y)=\sigma_{t}^{\psi}(y)(\forall y \in N, \forall t \in \mathbf{R})$, then $e_{\varphi, \psi}=1$.

Proof. (ii) By [3], there exists a unique n.f.s. operator valued weight $F: M_{+} \rightarrow \hat{N}_{+}$such that $\varphi=\psi \circ F$. For any $a \in n_{F}$, we have

$$
\varphi\left(y^{*} a^{*} a y\right)=\psi\left(F\left(y^{*} a^{*} a y\right)\right)=\psi\left(y^{*} F\left(a^{*} a\right) y\right) \leq\left\|F\left(a^{*} a\right)\right\| \psi\left(y^{*} y\right) \quad(\forall y \in N) .
$$

Hence $\mathfrak{n}_{F} \subset \mathfrak{N}$, this implies $e_{\varphi, \psi}=1$.

The weight $\varphi$ is said to be $\psi$-absolutely continuous in [4] if $M=N, \varphi+\psi$ is semifinite, and $\operatorname{Ker}(D \psi ; D(\varphi+\psi))_{-i / 2}=0$. The next Proposition shows that if $M=N$ and $M$ is a factor, then $e_{\varphi, \psi}=1$ is equivalent to $\varphi \leq \lambda \psi$ for some $\lambda>0$.

Proposition 1.3. If $M=N$ and $e_{\varphi, \psi}=1$, then the following statements are satisfied.

(i) $\varphi+\psi$ is an n.f.s. weight.

(ii) $\varphi$ is $\psi$-absolutely continuous.

(iii) If $M$ is a factor, then there exists $\lambda>0$ such that $\varphi \leq \lambda \psi$.

Proof. (i) From Lemma 1.1, $\mathfrak{N} \mathfrak{n}_{\psi} \subset \mathfrak{n}_{\varphi} \cap \mathfrak{n}_{\psi}=\mathfrak{n}_{\varphi+\psi}$.

(ii) Let $d=(D \psi ; D(\varphi+\psi))_{-i / 2}$ and assume $d \xi=0, \xi \in \mathfrak{S}$. We choose a sequence $\left\{x_{n}\right\}_{n \in N} \subset n_{\varphi+\psi}$ such that $\lim _{n \rightarrow \infty} \eta_{\varphi+\psi}\left(x_{n}\right)=J \xi\left(J=J_{N}=J_{M}\right)$. Then

$$
\lim _{n \rightarrow \infty} \eta_{\psi}\left(x_{n}\right)=\lim _{n \rightarrow \infty} J d J \eta_{\varphi+\psi}\left(x_{n}\right)=J d \xi=0 .
$$

Since, if $a \in \mathfrak{N}$, there exists $\lambda>0$ such that $\left\|\eta_{\varphi}\left(a x_{n}\right)\right\|^{2} \leq \lambda\left\|\eta_{\psi}\left(x_{n}\right)\right\|^{2}$, we have that $\lim _{n \rightarrow \infty} \eta_{\varphi}\left(a x_{n}\right)=0$.

Consequently, for any $a \in \mathfrak{N}$,

$$
\begin{aligned}
\|a J \xi\|^{2} & =\lim _{n \rightarrow \infty}\left\|\eta_{\varphi+\psi}\left(a x_{n}\right)\right\|^{2} \\
& =\lim _{n \rightarrow \infty}\left\|\eta_{\varphi}\left(a x_{n}\right)\right\|^{2}+\lim _{n \rightarrow \infty}\left\|\eta_{\psi}\left(a x_{n}\right)\right\|^{2}=0 .
\end{aligned}
$$

Since $e_{\varphi, \psi}=1$, this implies $\xi=0$.

(iii) By (ii), $d$ has the inverse. Hence we have

$$
\eta_{\varphi+\psi}(y)=J d^{-1} J \eta_{\psi}(y) \quad\left(\forall y \in \mathfrak{n}_{\varphi+\psi}\right) .
$$


Let $f \in \mathfrak{N}$ be a nonzero projection. Then there exists a $\lambda>0$ such that

$$
\left\|f J d^{-1} J \eta_{\psi}(y)\right\|^{2}=\left\|\eta_{\varphi+\psi}(f y)\right\|^{2} \leq(1+\lambda)\left\|\eta_{\psi}(y)\right\|^{2} \quad\left(\forall y \in \pi_{\varphi+\psi}\right) .
$$

It follows from above that $f J d^{-1} J$ is bounded. Let $\left(J d^{-1} J\right)^{*}=v h$ be the polar decomposition, then $h$ is affiliated to $M^{\prime}$, for $J d^{-1} J$ is affiliated to $M^{\prime}$. Thus we have $h f \supset f h$. Moreover, from

$$
f h=f h v^{*} v=f J d^{-1} J v
$$

we have that $f h$ is bounded, so that $f h f(\supset f h)$ is bounded. Hence if $h=\int \mu d E_{\mu}$ is the spectral decomposition, then $\overline{f h f}=\int \mu d\left(f E_{\mu} f\right)$ and there exists $\mu_{0}>0$ such that $f\left(1-E_{\mu_{0}}\right) f=0$. On the other hand, since $M$ is a factor, the induction $x \in M^{\prime} \mapsto f x f \in f M^{\prime} f$ is isomorphic. Hence, $1-E_{\mu_{0}}=0$. This means that $h$ is bounded, so that $J d^{-1} J$ is bounded.

Consequently, there exists $\lambda>0$, such that

$$
\begin{aligned}
\varphi\left(y^{*} y\right) & \leq\left\|\eta_{\varphi+\psi}(y)\right\|^{2}=\left\|J d^{-1} J \eta_{\psi}(y)\right\|^{2} \\
& \leq \lambda\left\|\eta_{\psi}(y)\right\|^{2}=\lambda \psi\left(y^{*} y\right) \quad\left(\forall y \in n_{\varphi+\psi}\right) .
\end{aligned}
$$

Since $\mathfrak{N} \mathfrak{n}_{\psi} \subset \mathfrak{n}_{\varphi+\psi}$, it follows that $\varphi\left(y^{*} y\right) \leq \lambda \psi\left(y^{*} y\right)$ for any $y \in \mathfrak{n}_{\psi}$.

For each $a \in \mathfrak{N}$, we have a unique bounded linear map $\mathbb{V}_{a}: \mathfrak{A} \rightarrow \mathfrak{D}$ which satisfies

$$
V_{a} \eta_{\psi}(y)=\eta_{\varphi}(a y) \quad\left(\forall y \in \mathfrak{n}_{\psi}\right) .
$$

It is easy to see that

$$
x V_{a} y=V_{x a y} \quad(\forall x \in M, \forall y \in N, \forall a \in \mathbb{I}) .
$$

Lemma 1.4. If $a, b \in \mathfrak{N}$, then

$$
a^{*} a \leq b^{*} b \Rightarrow \omega_{J_{M} V_{a} \xi} \leq \omega_{J_{M} V_{b} \xi} \text { on } M \quad(\forall \xi \in \mathfrak{S}) \text {. }
$$

Proof. If $y \in \mathrm{n}_{\psi}$, then

$$
\omega_{J_{M} V_{a} \eta_{\psi}(y)}=\theta_{\varphi}\left(y^{*} a^{*} a y\right) \leq \theta_{\varphi}\left(y^{*} b^{*} b y\right)=\omega_{J_{M} V_{b} \eta_{\psi}(y)} .
$$

Since $\eta_{\psi}\left(n_{\psi}\right)$ is dense in $\Re$,

$$
\omega_{J_{M} V_{a} \xi} \leq \omega_{J_{M} V_{b} \xi}
$$

hold for any $\xi \in \mathfrak{R}$.

From the above Lemma, it is easy to see that if $a^{*} a=b^{*} b(a, b \in \mathfrak{I t})$, then $\omega_{J_{M} V_{a} \xi}=\omega_{J_{M} V_{b} \xi}$ on $M$ for any $\xi \in \mathfrak{A}$. Moreover we see that

$$
\left\{\omega_{J_{M} V_{a^{1 / 2}}} \in M_{*}^{+}: a \in \mathfrak{M} \cap M_{+},\|a\|<1\right\}
$$

is upward directed for any $\xi \in \Re$. 
For each $\xi \in \Re$, let $\varphi_{\xi}$ be a normal weight on $M$ such that

$$
\varphi_{\xi}(x)=\sup \left\{\omega_{J_{M} V_{a 1 / 2} J_{N} \xi}(x): a \in \mathfrak{M} \cap M_{+},\|a\|<1\right\} \quad\left(\forall x \in M_{+}\right) .
$$

Lemma 1.5. If $\xi, \eta \in \mathfrak{R}$, then

$$
\omega_{\xi}=\omega_{\eta} \text { on } N \Rightarrow \varphi_{\xi}=\varphi_{\eta} .
$$

Proof. Note that there exists a partial isometry $u^{\prime} \in N^{\prime}$ such that $u^{\prime} \xi=\eta$.

Indeed, we can define $u^{\prime}$ by $u^{\prime} y \xi=y \eta$ for $\forall y \in N$ and $u^{\prime} \zeta=0$ for $\forall \zeta \in[N \xi]^{\perp}$.

Let $u=J_{N} u^{\prime} J_{N} \in N$. Then for any $a \in \mathfrak{M} \cap M_{+},\|a\|<1$, we have

$$
\omega_{J_{M} V_{a^{1 / 2}} J_{N} \eta}=\omega_{J_{M} V_{a^{1 / 2}} u J_{N} \xi}=\omega_{J_{M} V_{a^{1 / 2} u^{J_{N}} \xi}}=\omega_{J_{M} V_{\left(u^{*} a u\right)^{1 / 2}} J_{N} \xi} \leq \varphi_{\xi}
$$

Hence, $\varphi_{\eta} \leq \varphi_{\xi}$, so that $\varphi_{\eta}=\varphi_{\xi}$.

For any $\omega \in N_{*}^{+}$, there exists a $\xi \in \Re$ such that $\omega=\omega_{\xi}$. We shall put

$$
\varphi_{\omega}=\varphi_{\xi} .
$$

By the above Lemma, this definition does not depend on the choice of $\xi$.

Lemma 1.6. For any $x \in M_{+}$, there exists an element $E(x) \in \hat{N}_{+}$such that

$$
\langle E(x), \omega\rangle=\left\langle x, \varphi_{\omega}\right\rangle \quad\left(\forall \omega \in N_{*}^{+}\right) .
$$

Proof. Since for any $x \in M_{+}$and $a \in \mathfrak{M} \cap M_{+}$the map

$$
\xi \in \mathfrak{S} \mapsto \omega_{J_{M} V_{a^{1 / 2}} J_{N} \xi}(x)=\left\|x^{1 / 2} J_{M} V_{a^{1 / 2}} J_{N} \xi\right\|^{2}
$$

is continuous, it follows that

$$
\xi \in \mathfrak{R} \mapsto \varphi_{\xi}(x)=\sup \left\{\omega_{J_{M} V_{a^{1 / 2}} J_{N} \xi}(x) ; a \in \mathfrak{M} \cap M_{+},\|a\|<1\right\}
$$

is a lower semicontinuous positive quadratic form. Hence there exists a positive operator $h$ on $\Omega$ such that $\left\|h^{1 / 2} \xi\right\|^{2}=\varphi_{\xi}(x) \forall \xi \in \Re$.

Since for any unitary $u^{\prime} \in N^{\prime}$, we have

$$
\omega_{J_{M} V_{a^{1 / 2}} J_{N} u^{\prime} \xi}=\omega_{J_{M} V_{a^{1 / 2}} u J_{N} \xi}=\omega_{J_{M} V_{\left(u^{*} a u\right)^{1 / 2}} J_{N} \xi}
$$

where $u=J_{N} u^{\prime} J_{N}$, it follows that $\varphi_{u^{\prime} \xi}=\varphi_{\xi}(\forall \xi \in \mathfrak{A})$. Therefore $h \equiv E(x)$ is affiliated to $N$.

Theorem 1.7. For given $\varphi$ and $\psi$, there exists a normal $N$-weight $E: M_{+} \rightarrow$ $\hat{N}_{+}$such that

(i) $\left\langle E(x), \theta_{\psi}(y)\right\rangle=\left\langle\theta_{\varphi}(x), y e_{\varphi, \psi}\right\rangle \quad\left(\forall x \in \mathfrak{m}_{\varphi} \cap M_{+}, \forall y \in \mathfrak{m}_{\psi} \cap N_{+}\right)$,

(ii) $\psi \circ E=\sup \left\{\varphi_{\theta_{\psi}(y)} ; y \in \mathfrak{m}_{\psi} \cap N_{+},\|y\|<1\right\} \leq \varphi$.

Proof. (i) Let $E(x)$ be as in Lemma 1.6. Then it is easy to see that $E$ is a normal $N$-weight on $M$. For any $x \in \mathrm{m}_{\varphi} \cap M_{+}$and $y \in \mathrm{m}_{\psi} \cap N_{+}$, we have 


$$
\begin{aligned}
\varphi_{\theta_{\psi}(y)}(x) & =\sup \left\{\omega_{J_{M} V_{a^{1 / 2}} \eta_{\psi}\left(y^{1 / 2}\right)}(x) ; a \in \mathfrak{M} \cap M_{+},\|a\|<1\right\} \\
& =\sup \left\{\left\langle x, \theta_{\varphi}\left(y^{1 / 2} a y^{1 / 2}\right)\right\rangle ; a \in \mathfrak{M} \cap M_{+},\|a\|<1\right\} \\
& =\sup \left\{\left\langle\theta_{\varphi}(x), y^{1 / 2} a y^{1 / 2}\right\rangle ; a \in \mathfrak{M} \cap M_{+},\|a\|<1\right\} \\
& =\left\langle\theta_{\varphi}(x), y^{1 / 2} e_{\varphi, \psi} y^{1 / 2}\right\rangle=\left\langle\theta_{\varphi}(x), y e_{\varphi, \psi}\right\rangle .
\end{aligned}
$$

(ii) Since

$$
\psi=\sup \left\{\theta_{\psi}(y) ; y \in \mathrm{m}_{\psi} \cap N_{+},\|y\|<1\right\},
$$

it follows that

$$
\begin{aligned}
\psi \circ E & =\sup \left\{\theta_{\psi}(y) \circ E ; y \in \mathrm{m}_{\psi} \cap N_{+},\|y\|<1\right\} \\
& =\sup \left\{\varphi_{\theta_{\psi}(y)} ; y \in \mathrm{m}_{\psi} \cap N_{+},\|y\|<1\right\} .
\end{aligned}
$$

From (1), we have $\varphi_{\theta_{\psi}(y)} \leq \varphi\left(\forall y \in \mathfrak{m}_{\psi} \cap N_{+},\|y\|<1\right)$. Hence $\psi \circ E \leq \varphi$.

In the rest of this paper, $E$ will denote the map defined by Lemma 1.6.

Proposition 1.8. (i) If $\psi=\left.\varphi\right|_{N}$, then $E$ has a unique linear extension, which is a $\varphi$-conditional expectation.

(ii) If $e_{\varphi, \psi}=1$, then $\psi(E(x))=\varphi(x) \quad\left(\forall x \in m_{\varphi} \cap \mathbb{M}_{+}\right)$.

Proof. (i) Since $1 \in \mathfrak{N}$, it follows that $\varphi_{\omega} \in M_{*}^{+}\left(\forall \omega \in N_{*}^{+}\right)$. Hence $E(x) \in$ $N_{+}, \forall x \in M_{+}$. Therefore, $E$ has a unique linear extension from $M$ to $N$. If we also denote this map by $E$, then $E$ satisfies the following equation.

$$
\left\langle E(x), \theta_{\psi}(y)\right\rangle=\left\langle\theta_{\varphi}(x), y\right\rangle \quad\left(\forall x \in \mathfrak{m}_{\varphi}, \forall y \in \mathfrak{m}_{\psi}\right)
$$

(ii) For any $x \in m_{\varphi} \cap M_{+}$,

$$
\begin{aligned}
\psi(E(x)) & =\sup \left\{\left\langle E(x), \theta_{\psi}(y)\right\rangle ; y \in \mathrm{m}_{\psi} \cap N_{+},\|y\|<1\right\} \\
& =\sup \left\{\left\langle\theta_{\varphi}(x), y\right\rangle ; y \in \mathfrak{m}_{\psi} \cap N_{+},\|y\|<1\right\} \\
& =\left\langle\theta_{\varphi}(x), 1\right\rangle=\varphi(x) .
\end{aligned}
$$

\section{§2. The Case Where $\left.\sigma_{t}^{\varphi}\right|_{N}=\sigma_{t}^{\psi}, \forall \mathfrak{e} \in \mathbb{R}$}

In this section, we assume that $\varphi$ and $\psi$ satisfy

$$
\sigma_{t}^{\varphi}(y)=\sigma_{t}^{\psi}(y) \quad(\forall y \in N, \forall t \in \mathbb{R}) .
$$

Then by [3], there exists a unique n.f.s. operator valued weight $F: M_{+} \rightarrow \hat{N}_{+}$ such that $\varphi=\psi \circ F$. Hence, from Example 1.2, we have $e_{\varphi, \psi}=1$, in particular, $\mathfrak{N}=\mathfrak{n}_{F}$.

Lemma 2.1. The following equalities hold.

(i) $y J_{M} V_{a} J_{N} \zeta=J_{M} V_{a} J_{N} y \zeta \quad\left(\forall y \in N, \forall \zeta \in \mathfrak{A}, \forall a \in \mathfrak{N} \cap \pi_{\varphi}\right)$.

(ii) $\varphi_{y \omega y^{*}}=\varphi_{\omega}\left(y^{*} \cdot y\right) \quad\left(\forall \omega \in N_{*}^{+}, \forall y \in N\right)$. 
Proof. (i) Let $M_{a}^{\varphi}$ and $N_{a}^{\psi}$ be the sets of all entire analytic elements for $\varphi$ and $\psi$, respectively. Then from the assumption, we have that $N_{a}^{\psi} \subset M_{a}^{\varphi}$.

By [2, Lemma 7], we have that

$$
\eta_{\varphi}(a x)=J_{M} \sigma_{-i / 2}^{\varphi}\left(x^{*}\right) J_{M} \eta_{\varphi}(a) \quad\left(\forall a \in \mathrm{n}_{\varphi}, \forall x \in M_{a}^{\varphi}\right) .
$$

Hence, we get for $a \in \mathfrak{N} \cap \mathfrak{n}_{\varphi}, y \in N_{a}^{\psi}$ and $z \in N_{a}^{\psi} \cap \mathfrak{n}_{\psi}$

$$
\begin{aligned}
y J_{M} V_{a} \eta_{\psi}(z) & =y J_{M} \eta_{\varphi}(a z)=y \sigma_{-i / 2}^{\varphi}\left(z^{*}\right) J_{M} \eta_{\varphi}(a) \\
& =\sigma_{-i / 2}^{\varphi}\left(\sigma_{i / 2}^{\varphi}(y) z^{*}\right) J_{M} \eta_{\varphi}(a) \\
& =J_{M} \eta_{\varphi}\left(a z \sigma_{i / 2}^{\varphi}(y)^{*}\right) \\
& =J_{M} V_{a} \eta_{\psi}\left(z \sigma_{i / 2}^{\psi}(y)^{*}\right) \\
& =J_{M} V_{a} J_{N} y J_{N} \eta_{\psi}(z) .
\end{aligned}
$$

Since $J_{N} \eta_{\psi}\left(N_{a}^{\psi} \cap n_{\psi}\right)$ is dense in $\Re$ and $N_{a}^{\psi}$ is $\sigma$-weakly dense in $N$, we obtain (i).

(ii) Let $\left\{v_{i}\right\}_{i \in I} \subset \mathrm{n}_{\psi}$ be a net such that

$$
v_{i} \stackrel{s}{\rightarrow} 1
$$

Since for any $a \in \mathfrak{N}$, we have $a v_{i} \in \mathfrak{N} \cap \mathfrak{n}_{\varphi}$, using (i) we get

$$
\begin{aligned}
\omega_{y J_{M} V_{a} J_{N} \zeta} & =\lim _{i \rightarrow \infty} \omega_{y J_{M} V_{a v_{i}} J_{N} \zeta} \\
& =\lim _{i \rightarrow \infty} \omega_{J_{M} V_{a v_{2}} J_{N} y \zeta} \\
& =\omega_{J_{M} V_{a} J_{N} y \zeta} \quad(\forall \zeta \in \mathfrak{R}, \forall y \in N) .
\end{aligned}
$$

Thus, it follows that

$$
\varphi_{\zeta}\left(y^{*} x y\right)=\varphi_{y \zeta}(x) \quad \forall x \in M_{+} .
$$

Theorem 2.2. $E$ is equal to $F$.

Proof. By the above Lemma, we have

$$
\begin{aligned}
\left\langle E\left(y^{*} x y\right), \omega\right\rangle & =\left\langle y^{*} x y, \varphi_{\omega}\right\rangle=\left\langle x, \varphi_{y \omega y^{*}}\right\rangle \\
& =\left\langle y^{*} E(x) y, \omega\right\rangle \quad\left(\forall \omega \in N_{*}^{+}, \forall y \in N, \forall x \in M\right) .
\end{aligned}
$$

This means that $E$ is an operator valued weight. Hence to prove the theorem, it is sufficient to show that $\psi \circ E=\varphi$. [3]

We can obtain $b_{j} \in M_{a}^{\varphi} j \in J$ such that

$$
b_{j} \in \mathfrak{M} \cap M_{+}, \quad\left\|b_{j}\right\|<1, \quad \sigma_{\alpha}^{\varphi}\left(b_{j}\right) \stackrel{s^{*}}{\rightarrow} 1 \quad(\forall \alpha \in \mathrm{C}) .
$$

In fact, using some $a_{j} \in \mathfrak{M} \cap M_{+} j \in J$ which satisfies

$$
\left\|a_{j}\right\|<1 \quad(\forall j \in J) \quad \text { and } \quad a_{j} \stackrel{s^{*}}{\rightarrow} 1,
$$


we may define $b_{j}$ by

$$
b_{j}=\sqrt{\frac{1}{\pi}} \int_{-\infty}^{\infty} e^{-t^{2}} \sigma_{t}^{\varphi}\left(a_{j}\right) d t .
$$

Similarly, we can obtain $y_{k} \in N_{a}^{\psi}(k \in K)$ such that

$$
y_{k} \in \mathfrak{n}_{\psi} \cap \mathfrak{n}_{\psi}^{*}, \quad\left\|y_{k}\right\|<1 \quad(\forall k \in K), \quad \sigma_{\alpha}^{\psi}\left(y_{k}\right) \stackrel{s^{*}}{\rightarrow} 1 \quad(\forall \alpha \in \mathbb{C}) .
$$

Since $b_{j}$ and $y_{k}$ are analytic, we have that

$$
\begin{aligned}
J_{M} \mathbb{V}_{x b_{j}} \eta_{\psi}\left(y_{k}^{*}\right) & =J_{M} \eta_{\varphi}\left(x b_{j} y_{k}^{*}\right)=\sigma_{-i / 2}^{\varphi}\left(y_{k} b_{j}\right) J_{M} \eta_{\varphi}(x) \\
& =\sigma_{-i / 2}^{\psi}\left(y_{k}\right) \sigma_{-i / 2}^{\varphi}\left(b_{j}\right) J_{M} \eta_{\varphi}(x) \rightarrow J_{M} \eta_{\varphi}(x) \quad(j, k \rightarrow \infty)
\end{aligned}
$$

for any $x \in \mathfrak{n}_{\varphi}$.

Therefore, we have that for $x \in \mathfrak{n}_{\varphi},\|x\|<1$

$$
\begin{aligned}
\theta_{\varphi}\left(x^{*} x\right) & =\lim _{j, k \rightarrow \infty} \omega_{J_{M} V_{x b_{j}} \eta_{\psi}\left(y_{k}^{*}\right)} \\
& \leq \sup _{k} \varphi_{J_{N} \eta_{\psi}\left(y_{k}^{*}\right)}=\sup _{k} \varphi_{\theta_{\psi}\left(y_{k}^{*} y_{k}\right)} \\
& =\sup _{k} \theta_{\psi}\left(y_{k}^{*} y_{k}\right) \circ E \\
& \leq \psi \circ E \quad\left(\forall x \in \mathfrak{n}_{\varphi},\|x\|<1\right) .
\end{aligned}
$$

This implies $\varphi \leq \psi \circ E$. Therefore, using Theorem 1.7, we have $\varphi=\psi \circ \mathbb{E}$.

Proposition 2.3. We have that

$\mathfrak{M} \cap \mathrm{m}_{\varphi} \cap M_{+}=\left\{x \in \mathrm{m}_{\varphi} \cap M_{+} ;\right.$there exists $\lambda>0$ such that $\left.\left.\theta_{\varphi}(x)\right|_{N} \leq \lambda \psi\right\}$.

Proof. According to [6, proposition 2.17], it is true that for any $y \in N_{a}^{\psi} \cap$ $n_{\psi} \cap n_{\psi}^{*}$

$$
\psi\left(\sigma_{i / 2}^{\psi}(y) \sigma_{-i / 2}^{\psi}\left(y^{*}\right)\right)=\psi\left(y^{*} y\right) .
$$

Thus, if $a \in \mathrm{m}_{\varphi} \cap M_{+}$and there exists $\lambda>0$ such that $\left.\theta_{\varphi}(a)\right|_{N} \leq \lambda \psi$, then for any $y \in N_{a}^{\psi} \cap n_{\psi} \cap n_{\psi}^{*}$, we have

$$
\begin{aligned}
\varphi\left(y^{*} a y\right) & =\left\|\eta_{\varphi}\left(a^{1 / 2} y\right)\right\|^{2}=\left\|J_{M} \sigma_{-i / 2}^{\psi}\left(y^{*}\right) J_{M} \eta_{\varphi}\left(a^{1 / 2}\right)\right\|^{2} \\
& =\left\langle\sigma_{-i / 2}^{\psi}\left(y^{*}\right)^{*} \sigma_{-i / 2}^{\psi}\left(y^{*}\right), \theta_{\varphi}(a)\right\rangle \\
& \leq \lambda \psi\left(y^{*} y\right) .
\end{aligned}
$$

Using the density of $\eta_{\psi}\left(N_{a}^{\psi} \cap \mathfrak{n}_{\psi} \cap \mathfrak{n}_{\psi}^{*}\right)$ in $\eta_{\psi}\left(\mathfrak{n}_{\psi}\right)$, we have that $a \in \mathfrak{M} \cap \mathbb{M}_{+}$.

Conversely, we assume $a \in \mathfrak{M} \cap \mathfrak{m}_{\varphi} \cap M_{+}$, then for some $\lambda>0$

$$
\begin{aligned}
\left\langle y^{*} y, \theta_{\varphi}(a)\right\rangle & =\left\|J_{M} y J_{M} \eta_{\varphi}\left(a^{1 / 2}\right)\right\|^{2}=\left\|\eta_{\varphi}\left(a^{1 / 2} \sigma_{i / 2}^{\psi}(y)^{*}\right)\right\|^{2} \\
& \leq \lambda \psi\left(\sigma_{i / 2}^{\psi}(y) \sigma_{i / 2}^{\psi}(y)^{*}\right)=\lambda \psi\left(y^{*} y\right) \quad\left(\forall y \in N_{a}^{\psi} \cap n_{\psi} \cap n_{\psi}^{*}\right) .
\end{aligned}
$$

By the same argument as above, we have $\left.\theta_{\varphi}(a)\right|_{N} \leq \lambda \psi$. 


\section{References}

[1] Accardi, L., and Cecchini, C., Conditional expectations in von Neumann algebras and a theorem of Takesaki, J. Funct. Anal., 45 (1982), 245-273.

[2] Connes, A., Sur le théorème de Radon-Nikodym pour les poids normaux fidèles semifinis, Bull. Sci. Math., 97 (1973), 253-258.

[3] Haagerup, U., Operator valued weights in von Neumann algebras, I, II, J. Funct. Anal., 32 (1979), 175-206; 33 (1979), 399-361.

[4] Kosaki, H., Lebesgue decomposition of states on a von Neumann algebras, Amer. J. Math., 107 (1985), 697-735.

[5] Petz, D., A dual in von Neumann algebras, Quart. J. Math. Oxford, 35 (1984), 475-483.

[6] Strătilă, S., Modular theory in operator algebras, Editura Academiei and Abacus Press, 1981

[7] Takesaki, M., Conditional expectation in von Neumann algebras, J. Funct. Anal., 9 (1972), 306-321. 
